\title{
Nanoscratch test - A tool for evaluation of cohesive and adhesive properties of thin films and coatings
}

\section{J. Tomastik, R. Ctvrtlik}

Regional Centre of Advanced Technologies and Materials, Joint Laboratory of Optics of Palacky University and Institute of Physics of Academy of Sciences of the Czech Republic, Faculty of Science, Palacky University, 17. listopadu 12, 77146 Olomouc, Czech Republic

\begin{abstract}
Thin films and coatings play an essential role in the whole range of applications. The example par excellence are optical thin films that broaden the possibilities of design of optical components. Analogically to other applications of thin films their mechanical properties are very important for their successful applicability and reliability. This becomes especially vital when they are employed in rough service conditions. As thin films on substrates inherently create a compact system the strength of the film-substrate interface is of great importance. Several experimental methods have been developed for qualification and quantification of the mechanical stability of the film-substrate system. In this paper, some brief introduction into the nanoscratch test is introduced. It is currently the most widely used method to evaluate and to test cohesive-adhesive properties of thin films and coatings.
\end{abstract}

\section{Introduction}

Technology of thin films and coatings is nowadays an inherent feature of many industrial applications. Modern PVD (Physical Vapour Deposition) and CVD (Chemical Vapour Deposition) techniques offer the possibility to tailor thin films and coatings with various composition and physical and chemical properties. Application of coatings improves both performance and reliability of advanced engineering components, thereby saving the industry a great deal of capital. In the field of optical technology, single or multi-layered thin films can modify the optical properties of components to desired quality. Disc drives in computer industry, cutting tools and artificial joints also fully rely on special coatings and their performance [1,2].

There is logically a big demand for reliable assessment of mechanical properties of thin films. Originally, the mechanical durability of coatings had been easily assessed by a simple rule - the harder the better [3]. Later, several authors took into account also the elastic modulus $E$ of the film and proposed the $H / E$ ratio as a better ranking parameter of film wear resistance instead of hardness $H$ alone [4,5].

However, the durability of the coating is significantly influenced by its adhesion to the underlying substrate, which a simple measurement of the parameters $H$ and $E$ does not reflect. Therefore, it is necessary to use test methods which simulate in-service conditions more closely. A scratch test method fulfils such demands.

\section{The Scratch Test}

The scratch test is an instrumented complex technique for the assessment of both adhesive and cohesive properties and tribological characteristics of thin films.

During the scratch test a diamond indenter is straightforwardly pulled over the investigated surface at a constant velocity as can be seen from Figure 1 . The normal force is applied either in steps or more often continuously (usually linear increase). The latter case is called progressive load scratch test or ramped scratch test. The level of forces used for investigation of thin films usually ranges from several tens to hundreds of milinewtons while the corresponding displacements are typically from several nanometers to hundreds of nanometers [6]. The most commonly used type of indenter is the diamond conical Rockwell indenter. However, sharp indenters like Berkovich or cube-corner are also used in some special cases $[7,8]$.

The stress field generated under the moving indenter leads to the occurrence of different failure modes like coating detachment, through-thickness cracking, plastic deformation and cracking in the coating and/or substrate. These failure modes arise as a result of cohesion failure of coating and/or substrate or due to failure of coatingsubstrate adhesion. The goal of the test is to find so called critical loads $\left(L_{\mathrm{C}}\right)$ at which characteristic failures occur. These results then provide information about coating quality, its adhesive and cohesive properties and tribological resistance [9].

This is an Open Access article distributed under the terms of the Creative Commons Attribution License 2.0, which permits unrestricted use, distribution, and reproduction in any medium, provided the original work is properly cited. 


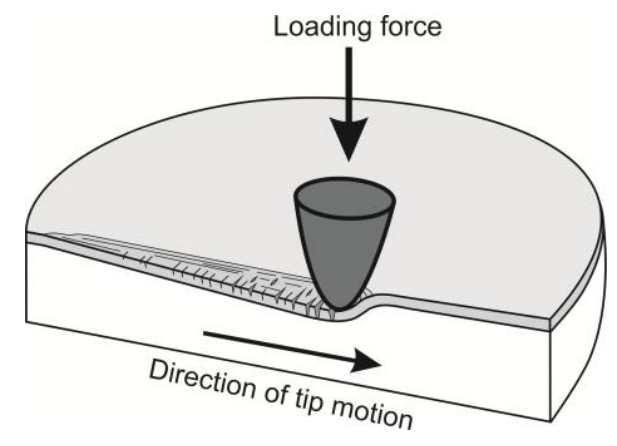

Fig. 1 Scratch test principle.

The typical course of the most often used progressive load scratch test is as follows: Before the test itself only minor force is loaded on the tip so it scans the surface. This data are used for surface roughness estimation and/or for compensation of the sample tilt. Then the load is gradually applied to the moving tip. At the beginning, surface deforms only elastically with no residual pattern on the surface. With increasing load the coating starts to deform plastically and the residual scratch on surface appears. Further increase of normal force results in creation of small cracks inside and at the edges of the scratch groove followed by more pronounced cracking and finally complete delamination of the coating. The onsets of failures, which appear regularly along the track, represent the critical loads, which can be considered as quantitative output of the scratch test method.

The nature of tribological contact of two surfaces in relative motion is very complex, which makes any simulation or prediction very difficult. Complexity of the system further increases if coating is introduced to the surface. Stress field resulting from the indenter movement can be understood as a sum of different contributions. Penetration of indenter into the surface cause bending of coating where both compressive and tensional stresses appear. The friction force between surface and sliding tip causes compressive stress in front of moving tip and tensile stress behind the tip. Another important factor is a magnitude of residual stresses stored in coating-substrate system during deposition process. Extensive analysis of these contributions can be seen in Holmberg [2].

\subsection{Critical load detection methods}

Detection of critical loads is primarily carried out by microscopic observation of residual scratch after the test. In addition there are three supporting detection techniques:

- Detection of sudden changes in penetration depth The stylus actual position controlled by sensitive depth sensor is usually recorded during the scratch test. Abrupt depth changes are then correlated to coating chipping or delamination.

- Friction probe - Coefficient of friction, defined as the ratio of the friction to normal force, can also be monitored during the test. The gradual increase of friction can be linked to ploughing while abrupt changes can be associated with fracture or catastrophic failure [10]. Friction force changes slightly even during the cracking of the coating [11].

- Acoustic emission probe - Acoustic emission signal arises from the sudden release of elastic energy. When coating cracks (even slightly) during the scratch test a shockwave is generated. These waves occur not only during total failure and detachment of the coating, but also in minor cracking at the coating-substrate interface or in the layer, which are poorly or not at all detectable by microscopic analysis. Stronger acoustic emission arises in cases of testing of hard coatings [12].

All these detecting methods become especially useful in cases where coating damage is poorly detectable by a microscopic investigation, particularly when coating is transparent.

\subsection{Adhesion assessment}

Application of the scratch test as a method for coating adhesion measurement were first mentioned in early 80 's by Perry [6,13], Steinman and Hintermann [14] and Valli [15]. Currently, the method is widely used in coating industry as well as in laboratory research. Nevertheless, some authors criticized its application for coatingsubstrate adhesion assessment (e.g. [16,17]). Although several failure modes are often observed during the scratch test evaluation, only few of them can be attributed to the detachment of the coating and thus directly correlated to adhesion. For example through thickness cracking is rather associated with cohesion strength of the coating. Furthermore, some failures occur simultaneously that makes the interpretation difficult.

\subsection{Typical failure modes}

The principal parameter predetermining response during the scratch test is considered coating and substrate hardness. In general, four possible situations can be distinguished:

- Soft coating on soft substrate - plastic deformation dominates and residual groove is created by ploughing. Crack formation is not present except at very high loads.

- Soft coating on hard substrate - plastic deformation dominates again. Coating gradually thins until the substrate is uncovered.

- Hard coating on soft substrate - deformation of substrate dominates. Coating bends into the track created by the plastic deformation of substrate. As a result, coating deforms plastically or cracks.

- Hard coating on hard substrate - Only minor or insignificant plastic deformation appears. Cracking of coating occurs, which can spread to the substrate [18].

Soft coatings generally tend to fail by ductile manner and are usually characterized by smaller cracks that are confined in the area of residual groove. In contrast, hard coatings are rather brittle and their cracks often extend beyond the edges of the track. 


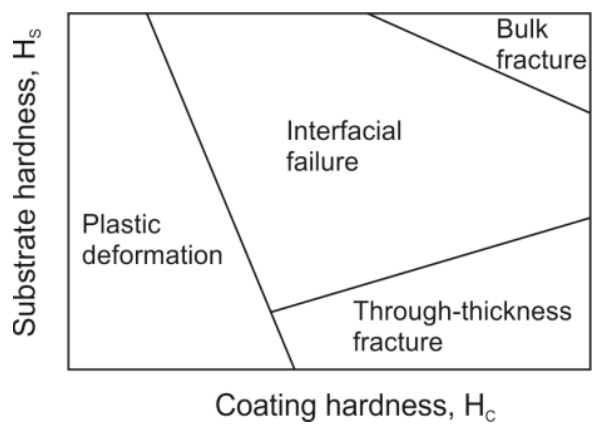

Fig. 2 Scratch test failures as a function of coating and substrate hardness [18].

Bull [19] described main brittle and ductile failure modes as depicted on Figure 3. Weak coating-substrate adhesion usually results in buckling or coating spallation. Coating could be stripped of along the entire length of scratch track if the adhesion is very poor. When strength of adhesion is higher than coating cohesion then different types of cracking occur. Minor cracking starts on the surface of coating and tends to spread through the coating up to the coating-substrate interface at higher loads. Fracture may even spread to the substrate depending on its mechanical properties. Failure may also arise due to local inhomogeneities.

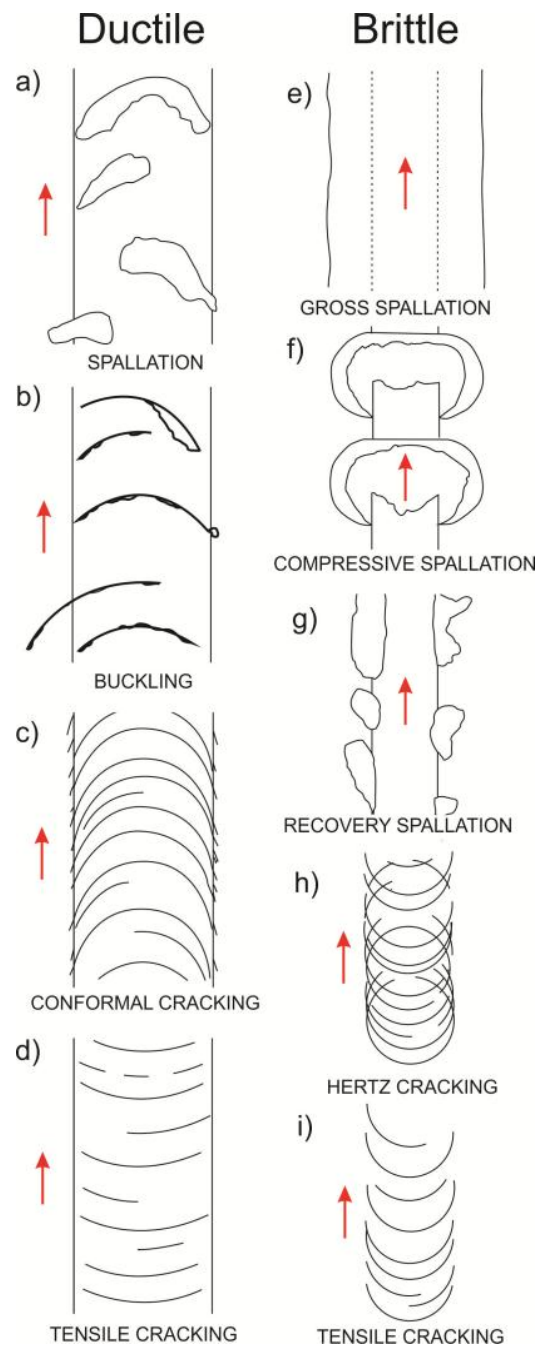

Fig. 3 Typical ductile and brittle failure modes [19].
Therefore, it is important to distinguish different types of failure modes and link them correctly with failure of adhesion or cohesion. It should be also noted that critical loads of various samples could be compared only for the same type of failure mechanism. Hence, there is a need for a thorough microscopic post-process analysis [18].

\subsection{Affecting parameters}

There are number of factors affecting the response of sample surface during the scratch test and hence the value of critical load. These factors can be divided into testbased intrinsic parameters and coating/substrate connected extrinsic parameters (Table 1). Their influence on critical loads was discussed in detail by Steinmann [20].

For instance critical load generally increases with increasing coating thickness and substrate hardness [6]. Choice of indenter shape and type is very significant as well. Blunt indenters have greater contact area with surface, which gives larger deformation area. Friction between the tip and the surface is more pronounced, thus changing the mechanism of failure production. On the other hand, sharper indenters have more localized contact with scratched surface; the deformation area is smaller and results are less affected by underlying substrate.

The comparison of results of the scratch test has to be done carefully. Values of critical load can be compared only in the case when all influencing factors remain constant [21].

Table 1. Parameters influencing the scratch test

\begin{tabular}{|l|l|}
\hline \multicolumn{1}{|c|}{ Intrinsic } & \multicolumn{1}{c|}{ Extrinsic } \\
\hline - Loading rate & - Substrate properties (hardness, \\
- Scratching speed & elastic modulus, coefficient of \\
- Indenter tip radius & thermal expansion) \\
- Indenter wear & - Coating properties (thickness, \\
- Machine compliance & hardness, modulus, residual \\
- External test conditions & stress) \\
(temperature, humidity) & - Friction coefficient \\
& - Surface roughness \\
\hline
\end{tabular}

\subsection{Multipass wear test}

Various modifications of the scratch test are also used [22]. The so called multipass wear test consists of multiple repetition of the simple scratch test over the same track; although the load is constant and lower then the critical load. Therefore, this test can be considered as a low cycle fatigue test. In fact, films usually have to withstand long-term or repetitive stresses rather than single extreme load impulse.

\section{Conclusions}

Material research as well as industry is in a great need for quick and precise instrumented methods for assessment of mechanical properties of coatings and their mechanical durability. The scratch test, as a suitable technique for assessment of adhesive and cohesive properties of thin 
coatings and their endurance, has been introduced. It allows investigating the coatings at various contact conditions by changing the experimental setup and simulating thus in-service conditions. A true comparison of various samples could be carried only if influencing factors are kept constant, in such situations the critical load which quantify the strength of adhesion could be compared over the set of samples. The scratch test is especially effective in the case of investigation of adhesion of hard and brittle coatings. Although the adhesion evaluation is much more difficult for softer coating, it is still applicable as a complementary test for other tests.

\section{Acknowledgement}

This work has been supported by the Operational Program Research and Development for Innovations European Regional Development Fund (CZ.1.05/2.1.00/03.0058) and co-financed from European Social Fund and State financial resources (CZ.1.07/2.3.00/20.0017), by Palacky University (internal grant no. PrF_2012_003) and by the Technology Agency of the Czech Republic (TA01010517).

\section{References}

1. D.S. Rickerby, Surf. Coat. Technol. 36, 541-557 (2004)

2. K. Holmberg, A. Laukkanen, H. Ronkainen, K. Wallin, S. Varjus, J. Koskinen, Surf. Coat. Technol. 200, 3793-3809 (2006)

3. J.F. Archard, J. Appl. Phys. 24, 981 (1953)

4. A. Leyland, A. Matthews, Wear 246, 1-11 (2000)

5. J. Musil, F. Kunc, H. Zeman, H. Poláková, Surf. Coat. Technol. 154, 304-313 (2002)

6. A.J. Perry, Thin Solid Films 107, 167-180 (1983)

7. V. Jardret, H. Zahouani, J.L. Loubet, T.G. Mathia, Wear 218, 8-14 (1998)

8. C.T. Pan, T.T. Wu, C.F. Liu, C.Y. Su, W.J. Wang, J.C. Huang, Mat. Sci. Eng. A 527, 2342-2349 (2010)

9. N.X. Randall, G. Favaro, C.H. Frankel, Surf. Coat. Technol. 137, 146-151 (2001)

10. B. Bushan, B.K. Gupta, Adv. Info. Storage Syst. 6, 193-208 (1995)

11. G. Subhash, W. Zhang, Wear. 252, 123-124 (2002)

12. J.H. Je, E. Gyarmatti, A. Naoumidis, Thin Solid Films 136, 57-67 (1986)

13. A.J. Perry, Thin Solid Films 78, 77-93 (1981)

14. P. Steinmann, H. Hintermann, J. Vac. Sci. Technol. A3, 2394-2400 (1985)

15. J. Valli, J. Vac. Sci. Technol. A3, 2411-2414 (1985)

16. J. von Stebut, R. Rezakhanlou, K. Anoun, H. Michel, M. Gantois, Thin Solid Films 181, 555-564 (1989)

17. J. Valli, J. Vac. Sci. Technol. A4, 3007-3014 (1986)

18. S.J. Bull, E.G. Berasetegui, Tribol. Int. 39, 99-114 (2006)

19. S.J. Bull, Surf. Coat. Technol. 50, 25-32 (1991)

20. P.A. Steinmann, Y. Tardy, H.E. Hintermann, Thin Solid Films 154, 333-349 (1987)

21. P.J. Burnett, D.S. Rickerby, Thin Solid Films 154, 403-416 (1987)
22. B.D. Beake, T.W. Liskiewicz, Tribol. Int., (in press, 2012) 